DER

\title{
LIBER CANCELLARIAE APOSTOLICAE VOM JAHRE 1380 \\ UND DER
}

\section{STILUS PALATII ABBREVIATUS DIETRICHS VON NIEHEIM.}

\author{
HERAUSGEGEBEN \\ VON \\ DR. GEORG ERLER, \\ PRIVATDOZENTEN AN DER UNIVERGITÄT LEIPZIQ.
}

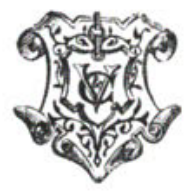

LEIPZIG, VERLAG VON VEIT \& COMP. 1888. 
Druck von Metzger \& Wittig in Leipzig. 


\section{DEM ANDENKEN}

\section{AN \\ WILHELM DIEKAMP.}

Gestorben zu Rom an 25. Dezember 1885.

Ich war im Herbst 1885 in der Bibliothèque nationale zu Paris mit der Abschrift des Liber cancellariae beschäftigt und hatte diese nahezu vollendet, als mir von befreundeter Seite die Kunde zuging, daß Wilhelm Diekamp dieselbe Handschrift bereits abgeschrieben habe und sie demnächst zu veröffentlichen gedenke. Sogleich stand ich von meiner Arbeit ab, und da ich früher den Stilus palatii abbreviatus Dietrichs von Nieheim zur Herausgabe vorbereitet hatte und es mir wünschenswert erschien, daß dieses Werk mit dem Liber cancellariae zusammen herausgegeben würde, so wandte ich mich an den damals in Rom weilenden Gelehrten und bot ihm meine Abschrift des Stilus palatii abbreviatus zur Benutzung an.

Dem Briefwechsel, der sich an dies Anerbieten knüpfte, sollte die erhoffte persönliche Bekanntschaft nicht folgen. Als ich im Dezember 1885 nach Rom kam, um dort meine Studien fortzusetzen, fand ich Diekamp bereits von der schweren Krankheit heimgesucht, von der zu genesen ihm nicht beschieden war. Wenige Tage nach meiner Ankunft stand ich unter den dunklen Cypressen des Campo santo dei Tedeschi an dem frischen Grabhügel, der das, was irdisch an ihm war, deckte.

Der der Wissenschaft allzufrüh entrissene hochbegabte Gelehrte hat einen Teil seiner rastlosen Thätigkeit der Erforschung des 
päpstlichen Kanzlei- und Urkundenwesens gewidmet. Rühmenswertes war von ihm geleistet worden und Größeres stand noch von ihm zu erwarten. Als ihn der Tod von hinnen rief, war er gerade mit einem Quellenwerk zur Geschichte der päpstlichen Kanzlei beschäftigt, welches neben dem Liber cancellariae und dem Stilus palatii abbreviatus unter andern auch den Liber diurnus und die Kanzleiregeln umfassen sollte.

Aus dem Nachlasse Diekamps wurde mir die Abschrift des Liber cancellariae übergeben. Sie hat mich bei der Herausgabe des Werkes wesentlich gefördert.

Indem ich mit dem Liber cancellariae wenigstens einen Teil dessen, was der Verstorbene geplant, der Öffentlichkeit übergebe, empfinde ich es als eine Pflicht der Dankbarkeit, diese Schrift dem Andenken des trefflichen Gelehrten zu widmen, dem es nicht vergönnt war, das Werk, das er selbst mit so vielen Hoffnungen begonnen hatte, zu Ende zu führen.

Leipzig, am 5. Januar 1888. 


\section{EINLEITUNG.}

Erhalten ist uns der Liber cancellariae in zwei Handschriften, die sich beide in der Pariser Nationalbibliothek befinden, den Codd. lat. 4169 und 4170. Da die letztere eine aus dem vorigen Jahrhundert datierende Abschrift der ersteren ist, so kommt für uns nur der Codex lat. 4169, das Original, in Betracht.

Derselbe, früher als Cod. Colbertinus 3317 und Codex regius 3918.4 bezeichnet, wird unseres Wissens zuerst von Baluze ${ }^{1}$ als vetustissimum registrum cancellariae apostolicae erwähnt. Im Handschriftenkatalog der Bibliothek ist er als Formula cancellariae apostolicae aufgeführt.

Zur Handschrift ist durchgehends dasselbe starke Pergament wie zu den päpstlichen Bullen verwandt worden. Die Blätter sind $30,7 \mathrm{~cm}$ hoch und $23 \mathrm{~cm}$ breit. Sie sind stark kalciniert, die rauhen Seiten sind gedunkelt, die glatten vielfach vergilbt, alle zeigen die Spuren häufiger Benutzung.

Man zählt im ganzen 14 Quaternionen zu je acht Blättern. Die Quaternionen sind am oberen Rande sämtlich, zum teil auch am unteren Rande des ersten Blattes mit römischen Ziffern I-XIIII bezeichnet. Ihre Aufeinanderfolge wird überdies durch die Custoden bestimmt, die teils von der Hand des Schreibers, teils von einer anderen Hand herrühren. Außerdem sind die Blätter am oberen Rande wohl gleichzeitig mit den römischen Ziffern I-CX numeriert. Die letzten vier Blätter haben von neuer Hand die Zahlen $111-$ 114 erhalten.

Der Handschrift voraus geht eine aus zwei Blättern bestehende Lage dünneren Pergaments. Das erste Blatt ist auf die Rückseite des vorderen Einbanddeckels festgeklebt. Das zweite Blatt zeigt die Spuren getilgter Schrift. Darauf findet sich die Notiz:

' Vitae papar. Aven. Paris 1693. I, 747; 938. Pertz, Archiv VII, 47. 
Invent. [?] IIIIxxXVIII (d. i. 98), also wohl die Signatur, welche die Handschrift in der päpstlichen Kanzlei trug. Mit Blatt 112 schloB die Handschrift ab, wie die unbeschriebene Rückseite dieses Blattes zeigt, welche ursprünglich an einen Einbanddeckel festgeklebt gewesen sein muß. Daran schliessen sich noeh zwei Lagen Pergament zu je zwei Blättern, von denen die erste an die zweite angeheftet ist. Auf der Rückseite des letzten Blattes liest man von einer Hand des 15. Jahrhunderts die Worte Cancellaria Romane ecclesie.

Der Einband in Pappschalen, die mit grünlichbraunem Leder überzogen sind und oben und unten mit je einem, an der Seite mit zwei Lederriemen zum Zusammenbinden versehen waren, ist alt, rührt aber aus einer späteren Zeit als die Handschrift selbst her.

So weit der Text des Liber cancellariae reicht, also bis auf Fol. $107^{\text {b }}$, sind auf jeder Seite $29-31$ zumeist blinde Linien gezogen, von denen die erste vielfach vom Schreiber nicht benutzt worden ist. Jede Seite weist links und rechts je zwei, nur wenig von einander entfernte senkrechte Linien auf. Für die wagerechten Linien waren, und zwar für den ganzen Quaternio am Rande Zirkelstiche gemacht, die zum Teil beim Beschneiden des Pergaments verschwunden sind. Die erste und die letzte sind immer durchgezogen, während die anderen in der Regel nur die senkrechten Abschlusslinien verbinden.

Auf manchen Blättern, wie $65,82,107$, gewahrt man senkrechte blinde Linien, welche, wie die Blätter 65 und 82 zeigen, am unteren Rande auf zwei wagerechte, $0,8 \mathrm{~cm}$ von einander entfernte parallele Linien stoßen. Das Pergament war, wie man aus dieser Art der Liniierung ersieht, zur Benutzung als Bulle vorbereitet gewesen und hatte nachträglich, in der Mitte senkrecht durchgeschnitten und dann wagerecht gefaltet, für die Handschrift Verwendung gefunden. Der Abstand der Linien ist durchgehend derselbe. Nur bei den Nachträgen, wie auf Blatt 107, ist mit Rücksicht auf den beschränkten Raum ein geringerer Abstand gewählt worden.

Durchschnittlich nimmt die Schrift auf jeder Seite einen Raum von $21,5 \mathrm{~cm}$ Höhe und $16 \mathrm{~cm}$ Breite ein, so daß ein ziemlich breiter Raum bleibt; der vielfach anderen Händen \%u Bemerkungen gedient hat.

Die Schrift ist schön, groß und deutlich. Sie gleicht der Schrift, die sich mit geringen individuellen Abweichungen auf 
allen Bullen der Kanzlei Urbans VI. und seiner nächsten Nachfolger findet.

Von Blatt $24^{\mathrm{b}}$ an beginnt ein etwas anderer Duktus, welcher die Buchstaben ein wenig schräger zur Zeile stellt und zwischen c und $t$ schärfer, als es auf den vorhergehenden Blättern geschieht, unterscheidet. Doch war es allem Anschein nach dieselbe Hand, welche den ganzen Codex schrieb.

An einzelnen Orten, wie Blatt 1, 25, 89, 100 und 103, finden sich zierlich ausgeführte Initialen, für welche der Schreiber unter Angabe des auszuführenden Buchstabens Raum lieB. Von Farben sind dabei gold, blau, rot und grün zur Anwendung gekommen. Der Maler nennt sich in der Initiale auf Blatt $89 \mathrm{~S}$. de Aquila. Es ist vermutlich der Skriptor Stephanus de Aquila, der eine Bulle Bonifacius' IX. vom 3. Juni 1391 für Dietrich mundierte. ${ }^{1}$

Für andere Initialen und sämtliche Überschriften, ja selbst für einzelne Stellen des T'extes, welche hervorgehoben werden sollten, ist Zinnober verwendet worden. Der Schreiber ließ regelmäßig für die Initialen und Überschriften Lücken. Wie diese auszufüllen waren, ist stets daneben in Kursive bemerkt. Den Raum, den der Schreiber für die Rubriken ließ, genügte nicht immer, so da $B$ der Rubrikator oft das Ende der vorhergehenden Zeile oder den Rand benutzen mußte. Der Rubrikator band sich übrigens für die Überschriften nicht immer an den ihm vorgeschriebenen Wortlaut. Hier und da hat er wohl eine Initiale nachzutragen vergessen, aber im allgemeinen ging er doch recht sorgfältig zu Werke. Ja, wiederholt hat er selbst Fehler des Textes verbessert.

Der SchluBsatz auf Blatt 107a legt es nahe, Dietrich von Nieheim für den Schreiber des Codex zu halten. Dem ist jedoch nicht so. Auf Blatt 23 (S. 42 dieser Ausgabe) lesen wir nämlich einen $\mathrm{Zu}$ satz von anderer Hand: Isti duo ultimi additi fuerunt per eundem papam Ioannem, ut mihi T. plena informacio facta fuit etc. Die Hand, welche diesen Zusatz schrieb, stimmt genau überein mit der Handschrift Dietrichs, wie sie in einem Briefe des Skriptors an die Stadt Dortmund erhalten ist. ${ }^{2}$ Hat nun Dietrich, wie ja auch schon aus dem Wortlaute mit großer Wahrscheinlichkeit hervorgeht, jenen Zusatz geschrieben, dann kann, den auffälligen Unterschieden der beiden Handschriften nach zu urteilen, die Abschrift des Liber

${ }^{1}$ Lindner in den Forschungen zur D. Gesch. XXI, 71. $\quad{ }^{2}$ Vom 25. Oktober 1408. Im Archiv der Stadt Dortmund. Vergleiche Lindner, Forsch. zur Dtschen. Gesch. XXI, $76 \mathrm{ff}$. 
cancellariae nicht von ihm herrühren. Wohl blieb noch die Möglichkeit, daß Dietrich in jenem Zusatz nicht den in der päpstlichen Kanzlei üblichen Duktus, in welchem der ganze Codex geschrieben ist, sondern seine individuelle Handschrift anwandte, daß also aus der Verschiedenheit der beiden Handschriften nicht mit Notwendigkeit auf verschiedene Schreiber zu schließen sein würde. Es war daher die Schrift des Liber cancellariae mit einer Bulle zu vergleichen, welche Dietrichs Namen auf dem Umbug als den des Ingrossators aufweist. Eine solche Bulle vom 4. Juli 1379 findet sich im K. K. Haus-, Hof- und Staatsarchiv zu Wien. Ein Vergleich ergab, da $B$ die genannte Urkunde von einer anderen Hand herrührt als die des Liber cancellariae. ${ }^{1}$ Es kann daher Dietrich den Liber nicht selbst mundiert haben.

Die Worte des Schlußsatzes sind demnach dahin zu verstehen, daß Dietrich das alte Kanzleibuch abschrieb, daß er aber dann die mühevolle und zeitraubende Reinschrift einem andern überlieB und sich mit der Kollation des von ihm übersichtlicher gestalteten und ergänzten Stoffes begnügte. Wohl als Kollationsvermerk hat er an das Ende mehrerer Absätze ein 'T gesetzt.

Geschrieben wurde der Liber cancellariae, der Subskription auf Blatt $106^{b}-107$ zu Folge, im April 1380. Dietrich bekleidete damals in der päpstlichen Kanzlei die Amter eines Scriptor litterarum apostolicarum und eines Abbreviators, in denen er, mehrfache Unterbrechungen allerdings abgerechnet, bis zum Ende seines Lebens geblieben ist. ${ }^{2}$

Der Zweck des Buches ist aus dem Inhalte leicht ersichtlich. Es enthält die wichtigsten Vorschriften für die Kanzleibeamten, die Formeln für die Eide, welche die neueintretenden Beamten zu leisten hatten, die Konstitutionen über die Pflichten der einzelnen Ämter, vor allem die Taxordnungen für die auszufertigenden Schreiben, lauter Bestimmungen, welche von Zeit zu Zeit durch Vorlesen in der Kanzlei eingeschärft werden sollten. An Stelle des alten, durch die Zeit abgenutzten Buches trat es, als das offizielle Handbuch,

${ }^{1}$ Nach gütiger Mitteilung des Herrn Staatsarehivar Dr. Winter v. 23 November 1887. ${ }^{2}$ Vergl. des Herausgebers Dietrich von Nieheim. Leipzig 1887. S. $26 \mathrm{ff}$. Zu den dort angegebenen Urkunden, auf welchen Dietrich als Skriptor genannt wird, ist nachzutragen: 1392 Juni 15. Datum Rome apud S. P. XVII. Kal. Iul. Pont. nostri (Bonif. IX.) Anno Tercio. Pro T. de Nyem Franciscus. Urkundenbuch der Stadt Hildesheim, herausg. von Doebner. Hildesheim 1886. II, 433. 
welches, mit AusschluB jedes andern, dem Gebrauche der Kanzlei dienen sollte. Sein Wortlaut entschied in zweifelhaften Fällen, nach der Gelöbnisformel des Kanzleibuches wurde auch der neu eintretende Beamte verpflichtet, und die in das Buch aufgenommenen kurzen Stücke aus den Evangelien dienten vermutlich dazu, bei der Vereidigung die Stelle der Bibel zu vertreten. ${ }^{1}$

Papst Urban VI. war aus der Kanzlei selbst hervorgegangen. Er hat derselben seine Aufmerksamkeit zugewendet, und er hatte guten Grund dazu, denn die Stürme der ersten Jahre seines Pontifikats hatten auch die Kanzlei schwer getroffen und zumal des größten Teils der alten geschulten Beamten beraubt.

Mit der Neueinrichtung der Kanzlei hängt vielleicht auch die Anlage des neuen Kanzleibuches zusammen. Wurde doch auch unter Urbans Pontifikat im Jahre 1388 auf Befehl des Bischof's Augustinus, Thesaurars des Papstes, von dem Presbyter Antonius Landus de Alberto der Liber censualis iurium camerae apostolicae abgeschrieben. ${ }^{2}$

Der Liber cancellariae ist, wie zahlreiche Nachträge und allerhand Zeichen am Rande und die vielfachen Spuren des Gebrauches beweisen, in der Kanzlei stark benutzt worden.

Besonderes Interesse erwecken die Einträge die sich am Schluß über Aufnahme und Vereidigung von Abbreviatoren, Skriptoren, Advokaten des sacrum palatium, Konsistorialadvokaten und Prokuratoren finden. Diese Einträge, welche für die Kenntnis des Kanzleipersonals aus der Zeit Urbans VI., Bonifacius' IX. und Innocenz' VII. von großer Wichtigkeit sind, beginnen mit dem 10. Oktober 1380 und reichen, freilich mit erheblichen Lücken, bis zum 28. März 1405. Es ist leicht möglich, daß das Verzeichnis der Kanzleibeamten durch Verstümmelung der Handschrift, durch Verlust einiger Blätter, in den Zustand der Verwirrung gekommen ist, in welchem es sich im Liber cancellariae befindet. Aber, selbst wenn es ursprünglich vollständiger war, in keinem Falle konnte es, wie die zahlreichen Mängel im Datum, die häufigen Korrekturen und die Unachtsamkeit gegen die Zeitfolge darthuen, als ein offizielles Verzeichnis jener Beamten und ihrer Vereidigung gelten. Es mögen Notizen sein, die sich der Regens der Kanzlei aus einem

${ }^{1}$ Lindner a. a. O. 73. $\quad{ }^{2}$ Nach einer Notiz auf einer Abschrift des Liber censuum S. Romanae ecelesiae a Cencio Camerario compositus. Rom. Bibl. Corsini Nr. 1041. 34. D. 6. 
Liber officialium oder einer matricula (S. 197) machte oder machen lieb.

Der letzte Eintrag, von einer Hand, die von den verschiedenen Händen, welche die Zusätze machten, abweicht, findet sich auf S. 213. Dort lesen wir eine Bemerkung persönlicher Art, wie sie sich sonst nie im Liber cancellariae findet, bei dem Namen des Auditors Nicolaus de Vivianis. Es heißt von demselben, daß er bis jetzt, bis zum Jahre 1417, noch an der römischen Kurie weile und derzeit Bischof von Spoleto sei.

In jenem Jahre war der Liber cancellariae apostolicae vom Jahre 1380 nicht mehr in der päpstlichen Kanzlei oder wenigstens nicht mehr in deren Gebrauch.

Unter den Kanzleiregeln Papst Johanns XXIII. findet sich und zwar aus dem Beginn seines Pontifikats - auch eine Konstitution für die in der Rota zu erledigenden Beneficialangelegenheiten. Dazu macht der Vicekanzler, Kardinal Johann von Ostia, die Bemerkung: Ita est in libro cancellariae. ${ }^{1}$ Dietrichs Liber war mithin nicht mehr in Wirksamkeit.

Wie die Handschrift ihren Weg nach Paris gefunden hat, läßt sich nicht nachweisen.

Bei der Wiedergabe der Handschrift hat sich der Herausgeber streng an die Orthographie der Vorlage gehalten. Nur ist im Gegensatze zu jener - was nicht immer in ihr geschieht - zwischen $\mathrm{u}$ und $\mathrm{v}$ unterschieden worden. In der Interpunktion wie in der Anwendung der großen Anfangsbuchstaben ist er dagegen selbständig verfahren.

Was in der Handschrift mit roter Farbe geschrieben worden ist, ist in der Ausgabe im Text durch gesperrten Druck hervorgehoben. In den Noten soll dagegen der gesperrte Druck auf wichtigere Zusätze der Handschrift aufmerksam machen.

Zusätze, welche bei offenbaren Lücken des Textes vom Herausgeber hinzugefügt worden sind, sind in eckige Klammern gesetzt. Schreibfehler sind im Text verbessert, die Fehler selbst aber in den Noten angegeben worden.

Der Inhalt der Handschrift ist folgender: Iuramentum domini vicecancellarii sancte Romanae ecclesiae, quod habet praestare domino papae. . . . . . . . 1

${ }^{1}$ Handschr. in Erfurt. St. Peter in Fol. 68 Blatt $103^{\text {b. }}$ 
Iuramentum, quod praestabunt domino vicecancellario eius domestici et alii familiares . . . . . . . . . . . 2

Antiquum iuramentum scriptorum, qui solebant distribuere litteras rescribendas cancellariae duntaxat . . . . . 3

Iuramentum rescribendarii de gratia . . . . . . . . . 4

Iuramentum rescribendarii de justitia . . . . . . . . . 5

Iuramentum auscultatorum et conputatorum litterarum de

iustitia in correctoria . . . . . . . . . . . . 6

Iuramentnm auscultantium in cancellaria . . . . . . . 6

$\begin{array}{ll}\text { Iuramentum lectorum litterarum in audientia contradictarum } & \mathbf{6}\end{array}$

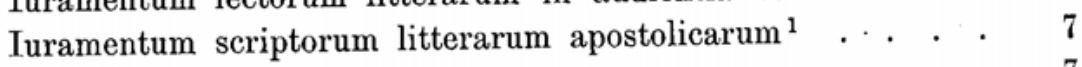

Iuramentum abbreviatorum . . . . . . . . . . . . . . 7

Iuramentum procuratorum audientiae contradictarum et advo-

catorum . . . . . . . . . . . . . . . . . 8

Additio reperta ex antiquis iuramentis cancellariae ad iura-

mentum procuratorum . . . . . . . . . . . . 8

Iuramentum auditorum palatii . . . . . . . . . . . . 9

Iuramentum notariorum palatii . . . . . . . . . . . 10

Iuramentum super tabellionatus officio . . . . . . . . 10

Iuramentum inquisitoris haereticae pravitatis $^{2}$. . . . . 11

Iuramentum abbreviatorum, qui tenent cameras dominorum

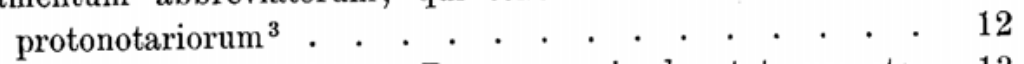

Iuramentum examinancium in Romana curia deputatorum etc. 13

Tituli examinatarum personarum etc. . . . . . . . . 13

Forma iuramenti poenitentiariorum . . . . . . . . . . 13

Initium evangelii secundum dominum Iohannem . . . . . 14

secundum Lucam . . . . . . . . . . . . . . . 15

Lectio sancti evangelii secundum Matthaeum . . . . . . 15

Lectio sancti evangelii secundum Marcum . . . . . . . 16

Lectio sancti evangelii secundum Lucam . . . . . . . 16

Sequuntur ordo et tituli dominorum cardinalium ecclesiae Ro-

manae sacrosanctae . . . . . . . . . . . . . 16

Destructio civitatis Penestrinae ac creatio seu erectio Civitatis

papalis . . . . . . . . . . . . . . . . . 18

Captationes speciales loco salutationis . . . . . . . . . 19

1 Gedr. Merkel, Documenta aliquot, quae ad Romani pontificis notarios et curiales pertinent im Arehiv. stor. Ital. Appendice V. Firenze 1847. S. 145.

2 Gedr. Lindner, Beiträge zu dem Leben und den Schriften Dietr. von N. in den

Forseh. zur Deutschen Geseh. XXI, 72 Ammerk. ${ }^{3}$ Gedr. Lindner a. a. O. 73. 
Provinciale

Supplik der Auditoren um die Erlaubnis einen tragbaren Altar besitzen und daran Messe lesen oder sich lesen lassen zu dürfen

Sammlung von häufig erteilten Privilegien, soweit sie vollständig oder in der Hauptsache angeführt werden, hier alphabetisch geordnet:

Animarum salutem desiderio ferventi quaerentes für die Cisterzienser . . . . . . . . . . . . . . . . . 80

Auctoritate vobis presentium für die Predigermönche . . . 109

Celestis amor patriae für die Predigermönche . . . . . 117

Christianae fidei religio für die Johanniter . . . . . . 67

Circa opera pietatis für St. Maria in Saxia . . . . . . 106

Circa opera pietatis fúr das Hospital de Altopassu . . . . 105

Cum abbates Cisterciensis ordinis für die Johanniter . . . 81

Cum a nobis petitur für die Predigermönche . . . . . . 114

Cum autem a religiosorum virorum pressuris für die Johanniter $\quad 97$

Cum Cisterciensis ordinis titulus für die Cisterzienser . . . 81

Cum de viris ecclesiasticis für die Templer . . . . . . 95

Cum dilecti filii für die Johanniter . . . . . . . . . 88

Cum dilectis filiis für dieselben. . . . . . . . . . . 83

Cum dilectorum filiorum für die Predigermönche . . . . 108

Cum iam per eius gratiam für dieselben . . . . . . . 110

Cum iam per eius gratiam für die Minoriten . . . . . . 120

Cum monachos et conversos für die Cisterzienser . . . $\quad 80$

Cum paupertatem et vitam für die Predigermönche . . . 107

Cum universis sanctae ecclesiae filiis für einen Erzbischof . 75

Decet pastoralis sollicitudinem für die Johanniter . . . . 99

Devotionis vestrae precibus inclinati für die Minoriten . . 121

Devotionis vestrae promeretur affectus für die Templer . . 96

Dilecti filii fratres Ierosolimitani für die Johanniter . . $\quad 89$

Dilecti filii fratres militiae templi für die Templer . . . . 86

Dilecti filii fratres militiae templi in provincia für dieselben 96

Dilecti filii fratres militiae templi post concilium für dieselben $\quad 96$

Etsi neque qui plantat für den Deutschen Orden . . . . 69

Evangelicae doctrinae, quae prohibet für die Johanniter . . 100

Ex parte vestra nobis est oblata für die Cisterzienser . . . 78

In eminenti apostolicae sedis specula für die Prämonstratenser $\quad 49$

In eminenti apostolicae sedis für einen Bischof . . . . . 74 
In iure canomico reperitur

In iure canonico reperitur für die Predigermönche . . . . 110

In iure canonico reperitur für die Minoriten . . . . . . 121

Inspirationis divinae gratia für Predigermönche . . . . . 112

Iustis petentium für die Johanniter . . . . . . . . . 98

Licet ad hoc fratrum für die Predigermönche . . . . . 113

Licet ad hoc fratrum für die Minoriten . . . . . . . . 123

Meminimus vobis olim für dieselben . . . . . . . . . 117

Milites templi Ierosolimitani für die Templer . . . . . . 92

Militia dei für dieselben . . . . . . . . . . . . . 94

Militum templi professio für dieselben . . . . . . . . 94

Ne pro eo, quod dyocessanis für die Predigermönche . . . 107

Non absque dolore cordis für die Cisterzienser, Templer u. a. 82

Non attendentes für die Minoriten . . . . . . . . . 123

Non solum in favorem conversi für die Predigermönche . . 118

Odore suavi ordinis vestrae für dieselben . . . . . . . 114

Omne datum optimum für die Templer . . . . . . . . . 62

Paci et quieti religiosorum virorum für dieselben . . . . 93

Pervenit ad nos ex conquestione für die Johanniter . . . 97

Postulastis a nobis für die Predigermönche . . . . . . 110

Precibus vestris benignum für dieselben . . . . . . . 117

Prohibente regula für die Minoriten . . . . . . . . . 122

Provisionis nostrae cupimus für die Predigermönche . . . 114

Quanto maiora pro defensione für die Johanniter . . . . 99

Quanto per gratiam dei für dieselben . . . . . . . . 100

Quanto studiosius divinae für die Cisterzienser . . . . . 79

Quanto studiosius divinae für dieselben, erweitert . . . . 80

Querela dilectorum filiorum für die Johanniter . . . . . 89

Querelam dilectorum filiorum für die Templer . . . . . 98

Querelam gravem recepimus für die Johanniter . . , . . 87

Querelam gravem recepimus für das Hosp. de Altopassu . . 104

Querelam gravem recepimus für das Hospit. von St. Spiritus in Saxia . . . . . . . . . . . . . . . . . 106

Quia confusio habitus für die Predigermönche . . . . . 111

Quia confusio habitus für dieselben an Erzbischöfe und Bischöfe . . . . . . . . . . . . . . . . . 111

Quia confusio habitus für die Minoriten . . . . . . . 122

Quia confusio habitus für dieselben an Erzbischöfe und Bischöfe 122

Quia refrigescente caritate für die Cisterzienser . . . . . 79

Quia proni sunt $a b$ adolescentia für die Predigermönche . . 112 
Qui deum totut seite

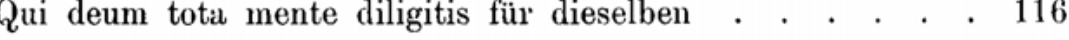

Quieti vestrae providere für die Minoriten . . . . . . . 120

Quieti vestrae providere für die Johanniter . . . . . . 101

Quieti vestrae providere für die Predigermönche . . . . . 108

Qui postposita vanitate für dieselben. . . . . . . . . 116

Qui postposita vanitate für die Minoriten . . . . . . . 124

Quoniam habundavit iniquitas für die Predigermönche. . . 108

Quo vos in Christo sinceriori caritate für dieselben . . . 119

Religiosam vitam eligentibus für die Cisterzienser . . . . 45

Religiosam vitam eligentibus für die Karthäuser . . . . 55

Religiosam vitam eligentibus für die Prämonstratenser . . . 57

Religiosam vitam eligentibus für die Clarissinnen . . . . 60

Religiosos viros für die Johanniter . . . . . . . . . 90

Sicut Iudaeis non debet esse licentia für die Juden . . . 77

$\mathrm{Si}$ diligenter attenditis für die Johanniter . . . . . . . 91

$\mathrm{Si}$ discrimina, quae dilecti filii für dieselben . . . . . . . 90

$\mathrm{Si}$ iuxta sententiam sapientis für das Hospital de Altopassu. 102

Si iuxta sententiam sapientis für das Hospital von St. Spiritus

in Saxia. . . . . . . . . . . . . . . . . 106

Vestra religio, cuius bonus odor für den Deutschen Orden . 101

Vestra semper in amore virtutum für die Predigermönche . 115

Vobis assidue regnum dei quaerentibus für dieselben . . . 115

Vobis extremam patientibus für dieselben . . . . . . . 113

Vobis extremam patientibus für die Minoriten . . . . . 124

Die den Cisterziensern gewährten Privilegien, nach den An-

fängen zusammengestellt . . . . . . . . . . . . . 59

Nachträge über Bewilligung der vorher im Wortlaut mitge-

teilten Privilegien an andere religiöse Gemeinschaften . 125

Aliquae constitutiones factae in concilio Lugdunensi ${ }^{1}$. . . 130

Aliae circa regimen vicecancellarii et notariorum constitutiones

cancellariae $^{2}$. . . . . . . . . . . . . . . 134

Forma rescriptorum introducta ex antiquo ${ }^{3}$. . . . . . 140

Exkommunikationsformel gegen solche, welche in Sachen einer

${ }^{1}$ Zum Teil gedr. Corp. iur. canon. Sext. Deeret. Lib. I. Tit. III cp. 2 ete. und bei Mansi, Sacr. concil. nova et ampl. collectio Venet. 1779. XXIII, $652 \mathrm{ff} .{ }^{2}$ Merkel $135-140 . \quad{ }^{3}$ Merkel $140-141$ unvollständig. Vollständig und mit dem Liber cancellariae, Umstellungen und verschiedene Lesarten abgerechnet, übereinstimmend: Pitra, Analecta noviss. spicileg. Solesmensis Typ. Tusculan. 1885. I, 162-167. 
iustititia oder gratia an der Kurie bestechen oder be-

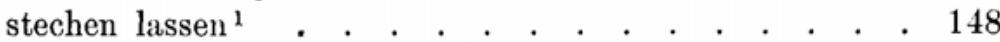

Einzelne Bestimmungen für die abbreviatores protonotariorum ${ }^{2} 148$ Sanctio gloriosissimi Frederici imperatoris pro ecclesiastica libertate $^{3}$. . . . . . . . . . . . . . . . 149

Constitutio [Honorii papae III.] contra exactionatores clericorum et dubios fidei catholicae ${ }^{4}$. . . . . . . . 152

Continuatio et denuntiatio constitutionis predictae . . . . 153

Forma dandi pallium . . . . . . . . . . . . . . 154

Forma iuramenti [archiepiscopi] . . . . . . . . . . . . . 155

Ista clausula debet apponi in litteris, quae mittuntur examinatoribus electionis . . . . . . . . . . . . . 155

Forma iuramenti, quod dominus Urbanus papa VI. ammodo voluit in litteris apostolicis super consecrationibus quorumlibet electorum conficiendis apponi scismate presenti durante . . . . . . . . . . . . . . . . . 156

Konstitution Johanns XXIII. Ratio iuris exigit ${ }^{5}$. . . . 157

Reservationes quorumdam beneficiorum factae per dominum

Iohannem vicesimum secundum anno sui pontificatus primo 167

Quaedam mandata dicti domini papae . . . . . . . . 168

Regimen et iura cancellariae more antiquo ${ }^{6}$. . . . . . 168

Quaedam constitutiones iuxta officium scriptoriae litterarum apostolicarum more antiquo . . . . . . . . . . 171

Konstitution Johanns XXII. Paterfamilias . . . . . . . 172

Konstitution Johanns XXII. Qui exacti temporis . . . . 191

Konstitution Benedicts XII. Decens et necessarium extimamus 196

Schlußbemerkung Dietrichs von Nieheim ${ }^{7}$. . . . . . . 203

Proklamation des Königs Karl von Neapel, erlassen im Lager vor Nocera gegen Papst Urban VI. Fol. 107. Gedr. Baluze, Vitae paparum Avinionen. II, 981.

Bestimmung Bonifacius' IX., erlassen in Sachen des Lütticher

1 Corp. iur. canon. Extravag. commun. Lib. V. Tit. X. De sententia excommun. und Merkel 144. $\quad{ }^{2}$ Merkel 145. $\quad{ }^{3}$ Mon. Germ. LI. II, 243. 245. ${ }^{4}$ Die noch nicht vollständig gedruckte Bulle Honorius III. vom 4. Januar 1221. M. G. EE. PP. I, 42. Dort an den Bischof von Bologna gerichtet. ${ }^{5}$ Vom 16. Nov. 1331. Gedr. Bullar. Roman. ed. Taurin. IV, 317-323. ${ }^{6}$ Gedr. bei Merkel 142-144 und Schlußsatz daselbst 146. ${ }^{7}$ Gedr. ein Bruchstück bei Baluze, Vitae pap. Avenion. I, 938. Vollst. bei Sauerland, Das Leben des Dietrich von Nieheim 73 und Lindner a. a. O. 71. 
Canonicus Wilhelm von Mokenborch an den Regens der päpstlichen Kanzlei Bartholomaeus Francisci . . . . 204 Bestimmung des Kardinalpresbyter Ramnulph, erlassen im Auftrage Papst Urbans VI. an Skriptoren und Abbreviatoren Entwurf zu einer Bulle, durch welche ein Anhänger des Gegenpapstes in die Obedienz Urbans VI. aufgenommen wird Fol. 108-110.

Nachträge über Vereidigung von Abbreviatoren, Skriptoren,

Konsistorialadvokaten etc. . . . . . . . . . . . 205

Nach der Darlegung des Inhalts erscheint es notwendig, die Zeit zu bestimmen, in welcher die einzelnen Teile des Liber cancellariae geschrieben oder zusammengestellt wurden.

Wir lassen den jüngsten Abschnitt, die Iuramenta der Kanzleibeamten, mit denen das Buch beginnt, bei Seite und wenden uns zuerst zu dem Verzeichnis der Kardinäle und der Übersicht über die Diöcesen der römischen Kirche. Beides zusammen faßte man frühzeitig unter dem Titel Provinciale cancellariae zusammen ${ }^{1}$, einer Bezeichnung, die in der Folge auch auf die mit ihm verbundene Privilegiensammlung und auf andere Aktenstücke, wie die Konstitutionen Johanns XXII. und Benedikts XII., überging, welche später ihren Platz hinter jener Sammlung in demselben Buche fanden. ${ }^{2}$

Den Kern der Liber cancellariae - denn diesen Namen trägt die ganze Sammlung bei Dietrich ${ }^{3}$ - bildet also das Verzeichnis der Kardinäle und der Diöcesen. Dieses stammt aus dem Beginn des 13. Jahrhunderts. Im Wesentlichen stimmt es überein mit dem Verzeichnis, welches Weidenbach aus einer Handschrift des 13. Jahrhunderts abdrucken lieB. ${ }^{4}$

Dieses ursprüngliche Verzeichnis ist trotz mannigfacher anderer Einteilung der Kirchensprengel nicht einer vollständigen Neubearbeitung unterzogen worden. Es begnügte sich vielmehr der Schreiber mit der Abschrift seiner mit vielen Nachträgen versehenen Vorlage, und er ließ dabei die Nachträge in der Form, in

${ }^{1}$ Vgl. S. 147 mit Beziehung auf die Privilegien: Item de hijs, que continentur in provinciali. ${ }^{2}$ Vgl. S. 4 und 5 in Bezug auf die Taxen in Johanns XXII. Konstitutionen: ordinatas, scriptas et insertas in provinciali cancellarie. ${ }^{3}$ S. 203; Finis unius libri cancellarie und S. 204: in presenti libro cancellarie. ${ }^{4}$ Calendarium historico-christianum medii ct novi aevi. Regensburg 1855. S. $223 \mathrm{ff}$. und $264 \mathrm{ff}$. 
welcher sie dem ursprünglichen Verzeichnis hinzugefügt worden waren, bestehen, denn es mochte nicht ohne Wert sein, die im Laufe der Jahre getroffenen Veränderungen der Diöcesaneinteilung und die Zeit der Gründung einzelner neuer Bistümer rasch übersehen zu können.

Von den Zusätzen, welche einen Schluß auf das Alter der ursprünglichen Zusammenstellung gestatten, sind nun folgende zu nennen:

S. 27 zu Rigensem sive Livonensem: qui hodie est archiepiscopatus per se. Darunter: Archiepiscopatus Rigensis fuit per dominum Alexandrum papam IIII. primo anno sui pontificatus de novo creatus etc. [1255].

S. 30: Appamiarum, qui fuit de novo creatus per dominum Bonifacium papam VIII. [16. IX. 1295].

S. 25: Sibenicensem. Et hic fuit de novo creatus per dominum Bonifacium papam VIII. [1. V. 1298].

S. 42: Archiepiscopatus Cambaliensis in dominio Tartarorum fuit creatus de novo per dominum Clementem papam V. [1307].

S. 29 und 30 folgen die zahlreichen neuen Bistümer, welche Johann XXII. in Frankreich gründete.

S. 42: Archiepiscopatus Soltaniensis in dominio Tartarorum parcium Aquilonarum fuit creatus de novo per felicis recordacionis Iohannem papam XXII. [1318].

S. 43: Archiepiscopatus Pragensis, qui de provincia Maguntina fuit subtractus, erectus est in ecclesiam metropolitanam etc. [30. IV. 1344].

Daneben finden sich noch zahlreiche Zusätze von anderer Hand:

S. 23: Caraciensem [Caiaciensem, Cajazzo, seit dem 8. Jahrhundert].

S. 23: Sagonensem [seit 1123].

S. 42: Satrensem alias Sitrensem [Sitiensem seit 1225].

S. 23: Naulensem [seit 1239].

S. 31: Archiepiscopatus Ispalensis mit seinen Diöcesen [seit 1248].

S. 42: Calamonensem [seit 1287].

S. 22: Nicotirensem erectum de novo [seit 1297].

S. 21: Sancti Leonis [seit 1322].

S. 20: Cortonensis. Dazu am Rande: Dominus Iohamnes papa XXII. in diocesi Aretina fecit alium episcopatum, qui vocatur Cortonensis [seit 1325].

S. 23: Bretonoriensem, d. h. Bertinoro. Hierher wurde das Bistum von Forlimpopoli verlegt [1360].

S. 20: Montisflasconis [seit 1369]. 
Eine ziemlich große Anzahl von Zusätzen ist also von anderen Händen nachgetragen worden.

Da nun für den Liber cancellariae als Vorlage ein offizielles Handbuch diente und wir nicht annehmen dürfen, daß jenes, soweit es sich wenigstens um abendländische Bistümer handelte, deren Inhaber in lebhaftem Verkehr mit Rom standen, mangelhafte Angaben enthielt, so daß sich also eine Korrektur oder Ergänzung aus andern Büchern notwendig gemacht hätte, so bleibt nur die Vermutung übrig, daB der Abschreiber manche Zusätze im alten Provinciale übersah und daß sich daher deren Nachtragung bei der Kollation als notwendig herausstellte.

Aus den Zusätzen von anderer Hand läßt sich ein Schluß auf das Alter der ersten Zusammenstellung des Diöcesanverzeichnisses nicht mit Sicherheit ziehen. Leicht konnte ja bei dem ältesten Provinciale ein Namen übergangen werden, den man später, als man den Mangel bemerkte, nachtrug. Ein solches Übersehen konnte dann namentlich Platz greifen, wenn es sich um unbedeutende Diöcesen handelte, wie die von Calatia und Sagona. Unmöglich aber erscheint es, daß die Erzdiöcese Sevilla, die mit ihren Suffragandiöcesen nach Zurückdrängung der Mauren hergestellt wurde, übergangen werden konnte. Die erste Zusammenstellung des Provinciale muß also vor der Mitte des 13. Jahrhunderts stattgefunden haben. Und auf diese Zeit weist auch der älteste umständlichere Nachtrag hin, den der Schreiber des neuen Liber cancellariae bezüglich des im Jahre 1255 neu gegründeten Erzbistum Riga aus seiner Vorlage herübernahm.

Außer diesen Nachträgen, welche der Ergänzung dienten, sind noch zwei Zusätze zu dem Provinciale gemacht worden. Der erste (S. 18), Destructio civitatis Penestrinae etc., wurde unterm Pontifikat Bonifacius VIII. im Jahre 1299 hinzugefügt. Der zweite, überschrieben Captationes speciales loco salutationis (S. 19), erwähnt den griechischen Kaiser Michael Paläologus und Kolomann, den Herrscher Bulgariens. Der erstere regierte 1260-1282. Bezüglich des letzteren läßt sich eine Entscheidung über die Zeit des Eintrags in das Provinciale nicht mit Sicherheit treffen. Es kann Kolomann I. (1241-1245) oder der zweite Herrscher dieses Namens (von $1258 \mathrm{ab})$ gemeint sein. Die Verbindung, in welcher der Name mit dem Paläologen auftritt, spricht für Kolomann II. Der Nachtrag rührt also aus der zweiten Hälfte des 13. Jahrhunderts her und ist jedenfalls vor 1282 eingefügt worden. 
Dem Provinciale folgt eine Supplik der Auditoren des sacrum palatium. Auch sie hat später erst hier ihren Plat\% gefunden, denn sie gehört, wie die Erwähnung des Konzils von Vienne und der Ort, an dem sie ausgestellt ist - Avignon -, beweisen, dem 14. Jahrhundert an.

Einen nicht unwichtigen Teil des Liber cancellariae bildet die Sammlung von Privilegien, welche mit dem Provinciale vereinigt ist und frühzeitig mit unter dem Namen desselben begriffen wurde.

Diese Sammlung hatte gewiB nicht den Zweck als Formelsammlung bei der Abfassung von Privilegien zu dienen, sondern wurde vermutlich dann erst zu Rate gezogen, wẻnn über den Wortlaut eines der Privilegien die Meinungen geteilt waren.

Die Sammlung ist nicht allmählich entstanden, sondern ist, wie wir gleich vorausschicken wollen, nach einem bestimmten Plane unterm Pontifikat Innocenz' IV. angelegt und teils unter jenem noch, teils unter seinen nächsten Nachfolgern um mehrere Zusätze vermehrt worden.

Sie beginnt mit dem Privilegium der Cisterzienser: Religiosam vitam eligentibus. Die Form, in welcher dasselbe erscheint, ist die erweiterte der späteren Zeit, wie sie sich am Ende des 12. Jahrhunderts entwickelte.

Das Privilegium Papst Cölestins III. für das Kloster Salem rom 6. Nov. 1194 [Weech, Cod. dipl. Salem. I, 81] stimmt schon fast ganz mit dem Privileg des Liber cancellariae überein. Nur fehlt hier noch der Zusatz ,ante concilium generale susceptam", und der Sat\% über den Zehnten, der ursprünglich: „Sane laborum vestrorum, quos propriis manibus aut sumptibus colitis, sive de nutrimentis vestrorum animalium nullus omnino a vobis decimas presumat exigere" lautete ${ }^{1}$, und später, wie in dem soeben erwähnten Privileg Cölestins III., erweitert wurde zu: „Sane laborum vestrorum, quos propriis manibus aut sumptibus colitis, tam de terris cultis quam incultis, sive de ortis et virgultis et piscationibus vestris vel de nutrimentis animalium vestrorum, nullus a vobis decimas exigere vel extorquere praesumat" ${ }^{2}$ hat im Liber cancellariae wieder eine

${ }^{1}$ Vgl. Alexander III. am 28. Juli 1171 für Morimund |Bull. Roman. ed. Taurin. II, 752]; am 4. Jan. 1178 für Salem [Weech, Cod. dipl. Salem. I, 35]. 2 Vgl. Papst Lucius III. am 4. März 1185 für Salem |Weech, Cod. dipl. Salemit. I, 52]; Innocenz III. vom 8. Nov. 1205 für Walkenried [Urk. des Stifters Walkenried I, 47]; voin 23. März 1214 fïr Alderspach |Bull. Roman. III, 292!. 
Wandlung erfahren zu: „Sane laborum vestrorum de possessionibus habitis ante concilium generale ac etiam novalium, quae propriis manibus aut sumptibus colitis, de quibus novalibus aliquis hactenus non percepit, sive de ortis virgultis et piscationibus vestris vel de nutrimentis animalium vestrorum, nullus a vobis decimas exigere vel extorquere presumat".

$\mathrm{Ob}$ man die Zehntfreiheit für alle Klostergüter oder nur für die Neubrüche zugestehen sollte, darüber haben verschiedene Ansichten gegolten. Während man früher die Zehntfreiheit auf alle Güter ausgedehnt hatte, beschränkte sie Hadrian IV. ${ }^{1}$ wegen der für die Kirche ungünstigen wirtschaftlichen Folgen auf die Neubrüche und ersetzte daher in seinen Privilegien das Sane laborum durch Sane novalium. Nach Hadrians Tod wurde der Gebrauch schwankend und verlieh man die Zehntfreiheit bald nur für Neubrüche, bald in vollem Umfange.

Im Prinzip geregelt wurde die Frage wohl erst von Innocenz III. auf dem Lateranischen Konzil von 1215. ${ }^{2}$ Seit jener Zeit herrschte der Gebrauch, dass die Zehntfreiheit, falls die Cisterzienserregel erst nach dem Konzil angenommen worden war, nur für die Neubrüche galt. Im anderen Falle wurde die Zehntfreiheit für alle Klosterwirtschaften erteilt, doch nur unter der Bedingung, daß die schon bewirtschafteten Güter vor dem Konzil in den Besitz des Klosters gekommen waren und die Neubrüche bisher keinen Zehnten gezahlt hatten. ${ }^{3}$

Genau in der Form, in welcher das Privilegium im Liber cancellariae erscheint, ist es von Gregor IX. am 21. Mai 1237 dem Kloster Schönthal und von Iunocenz IV. am 7. Juli 1250 dem Kloster Salem verliehen worden. ${ }^{4}$

Dem Privilegium der Cisterzienser folgt S. 49 das der Prämonstratenser In eminenti apostolicae sedis specula. Dasselbe ist gleichlautend mit dem von Innocenz III. am 27. Juli 1198 allen Äbten von Prämonstratenserklöstern erteilten Privileg. ${ }^{5}$ Nur ist die dort

1 Vgl. Maassen, Paucapalea. Sitz.-Ber. der Wiener Ak. Phil.-hist. Klasse 1859. XXXI, 469; Thaner, Entstehung der Formel: salva sedis apostolicae auctoritate das. 1872. LXXI, 828. ${ }^{2}$ Corp. iur. can. Decret. Gregor. IX. Lib. III. Tit. XXX. ep. 34. Vgl. Manrique, Annal. Cistere. IV, 62 u. die Bulle Honorius' III. voin 31. December 1218 (Bull. Roman. ed. 'Taurin. III, 347). ${ }^{3}$ Vgl. Honor. III. am 11. Okt. 1216 für Herrenalb. Wirtemb. Urkdb. III, 57; Gregor IX. am 8. März 1231 fïr Bebenhausen das. III, 252. Dagegen Beispiel für post concilium generale susceptam Gregor IX. am 20. Juni 1236 für Boos das. IU, 380. ${ }^{4}$ Wirtemb. Urkdb. III, 392; Weech, Cod. dipl. Salemit. I, 290. Bull. Roinan. III, 126. Vgl. Hertel, Urkundenbuch des Klosters Unsrer 
getroffene Bestimmung über die Zehntfreiheit: „Sane laborum vestrorum, quos propriis manibus aut sumptibus colitis, sive de nutrimentis vestrorum animalium nullus a vobis decimas exigere vel extorquere presumat, licet fundorum dominis pro rei proprietate aliquem censum vel quamlibet partem frugum reddatis" im Liber cancellariae genau nach der für das Cisterzienserprivilegium Religiosam vitam eligentibus gewählten Form umgewandelt worden.

Mit dem Cisterzienserprivilegium Religiosam vitam eligentibus stimmt auch das Karthäuserprivilegium Religiosam vitam (S. 55) eligentibus, soweit es sich um das Sane laborum handelt, überein.

Das auf S. 57 folgende Prämonstratenserprivilegium Religiosam vitam eligentibus kennt nur Zehntfreiheit für die Neubrüche und schreibt daher: Sane novalium. Es ist in dieser Form mit geringen Abweichungen von Innocenz IV. am 10. Mai 1249 erteilt worden. ${ }^{1}$

Das Privilegium für die Clarissinnen erwähnt die von Urban IV. am 18. Oktober 1263 gegebene Ordensregel ${ }^{2}$, muß also in dieser Form nach dem Tode des Papstes, also nach dem 2. Oktober 1264 entstanden sein. Eine solche Annahme würde der Ansicht freilich widersprechen, die wir im Anfange dieser Untersuchung aussprachen, daß nämlich die Privilegiensammlung unterm Pontifikat Innocenz' IV. zusammengestellt worden sei. Da aber Innocenz IV. am 9. August 1253 die erste Regel für die Clarissinnen gab, so ist es leicht möglich, daß ein Privilegium für diesen Orden, welches der von Innocenz IV. gegebenen Regel Erwähnung that, an dieser Stelle seinen Platz hatte, und später erst, nach Änderung der Regel durch Urban IV., der Name dieses Papstes eingesetzt wurde.

In dem Privilegium für den Templerorden (S. 62) Omne datum optimum etc. werden als Vorgänger des Ausstellers genannt: Alexander [III. 1159-1181], Lucius [III. 1181-1185; vgl. Prutz, Malteser Urk. und Reg. Nr. 20], Urban [III. 1185_-1187], Klemens [III. 1187-1191]. Mithin ist es nach 1191 geschrieben.

Das Privilegium für die Johanniter Christianae fidei religio (S. 67) nennt als Vorgänger des Ausstellers: Innocenz [II. 1130], Coelestin [II. 1143], Lucius [II. 1144], Eugen [III. 1145], Anastasius [IV. 1153], Adrian [III. 1154], Alexander [III.], Lucius [III.], Urban [III.], Gregor [VIII. 1187], Klemens [III.], Coelestinus [III. 1191-1198]. Es ist also nach 1198 abgefaßt.

lieben Frauen zu Magdeburg. Geschichtsquellen der Prov. Sachsen X, 72. ${ }^{1}$ Bull. Rom. III, 540. $\quad{ }^{2}$ Bull. Rom. III, 709. 
1)as für den Deutschen Orden an den Ordensmeister Hermann gerichtete Privilegium (S. 69) Etsi neque qui plantat ist das alte von Honorius III. am 15. Dezember 1220 erteilte. $^{1}$ Von Gregor IX. am 28. Juli 1227 wiederholt, ist es nur insofern verändert, als des ersten Ausstellers, Honorius' III., gedacht wird. ${ }^{2}$

S. 77 folgt das Privilegium Iudaeorum: Sicut Iudaeis. Es hat denselben Wortlaut wie die von Honorius III. am 7. November 1217 ausgestellte Urkunde. ${ }^{3}$ Da aber im Unterschied zu jener im Liber cancellariae Honorius III. als Vorgänger des Ausstellers genannt wird, so muB die Vorlage nach dem Tode des Papstes, also nach 1227 entstanden sein.

S. 81. Cum abbates Cisterciensis ordinis für die Johanniter, stimmt überein mit den für Walkenried ${ }^{4}$ und Salem ${ }^{5}$ von Innocenz IV. ausgestellten Privilegien. Der im Liber cancellariae als Vorgänger des Ausstellers genannte Imnocenz wäre demnach Innocenz III. In der That bezieht sich das Privileg auf die von Innocenz III. auf dem Lateranischen Konzil von 1215 über die Zehntfreiheit angeordnete Bestimmung: „Nuper abbates Cisterciensis ordinis“. ${ }^{6}$ Mithin stammt diese Vorlage des Liber cancellariae aus der Zeit nach 1216.

Von den anderen Privilegien geben noch folgende einen Anhalt zur Bestimmung der Zeit, in der sie verfaßt wurden.

S. 92 findet sich das Privilegium für die Johanniter Milites templi Ierosolimitani. Die Templer erhielten dasselbe von Eugen III. am 16. Juli $1145-46^{7}$, der deutsche Orden von Honorius III. am 16. Januar 1221. ${ }^{8}$ Der Liber cancellariae hat das Privilegium in der jüngeren Fassung, in der es Honorius III. dem deutschen Orden erteilte.

S. 93 folgt das Privilegium für die Templer Paci et quieti. Dasselbe rührt von Alexander III. her und wurde am 24. Februar 1160-61 ausgestellt. ${ }^{9}$

Das Privilegium für die Templer Militia dei, quae dicitur templi nennt Alexander als früheren Aussteller. Es ist dies Alexander III. Die Vorlage des Liber entstammt also der Zeit nach 1181. Der-

${ }^{1}$ Hennes, Cod dipl. ord. S. Mariae Theutonicon. Mainz 1845. S. ว̃o. Strehlke, Tabulae ordin. Theuton. Berol. 1869. Nr. 306. ${ }^{2}$ Bull. Rom. III, 423. ${ }^{3}$ Bull. Roman. III, 330. $\quad{ }^{4}$ Vom 1. März [1249]. Urkunden des Stiftes Walkenried S. $188 . \quad 5$ Vom 7. Okt. 1260. Weech, Cod. diplom. Salemit. I, 295. ${ }^{6}$ Decret. Gregor. IX. Lib. III. Tit. XXX. Cp. $34 .{ }^{7}$ Prut\%, Malteser Urkunden Nr. 1. ' Strehlke, Tabulae ordinis Theutonici Nr. 321. ${ }^{9}$ Prutz, Malteser Urkunden Nr. 4. 
selben Zeit gehört das Privilegium für die Johanniter an (S. 97), in welchem als früherer Aussteller ebenfalls Alexander [III.] erwähnt wird.

Das Privilegium für die Johanniter (S. 99) Quanto maiora pro defensione discrimina nennt als Vorgänger des Ausstellers: Alexander [III. 1159-1181], Lucius [III. 1181-1185] , Urban [III. 11851187], ist also nach 1187 enstanden. Das folgende Privilegium (S. 100) für denselben Orden Quanto per gratiam gedenkt des Ordensmeisters Raymund [de Puy] als eines Verstorbenen. Er starb 1160. Als Vorgänger des Ausstellers wird Eugen [III. 1145 -1153] genannt. Die Urkunde gehört somit der Zeit nach 1160 an.

Das an den Ordensmeister Hermann gerichtete Privileg des deutschen Ordens (S. 101) Vestra religio wurde zuerst von Honorius III. am 9. Januar 1221 gegeben. ${ }^{1}$ Nicht aber diese Urkunde, sondern - wie der Zusatz ad exemplar felicis recordacionis memoriae Honorii papae beweist - die gleichlautende seines Nachfolgers Gregor IX. vom 12. Juni $1227^{2}$ wurde in den Liber cancellariae aufgenommen.

S. $107 \mathrm{Ne}$ pro eo, quod dyocesanis für die Predigermönche wurde zuerst von Gregor IX. im Jahre 1227 gegeben ${ }^{3}$, wiederholt am 27. Nov. $1234^{4}$ und von Innocenz IV. am 4. September $1243 .{ }^{5}$

Cum paupertatem für die Predigermönche wurde zuerst von Honorius III. am 10. Februar 1221 erteilt. $^{6}$ Doch nicht in dieser Form gibt es der Liber cancellariae wieder, sondern in der erweiterten, in der es Innocenz IV. am 21. Januar 1244 nachmals verlieh. ${ }^{7}$

Cum tamquam veri et fideles für dieselben, zuerst von Gregor IX. am 9. April 1228 mit der Beschränkung ad opus fratrum et familiae vestrae gegeben. ${ }^{8}$ Der Liber cancellariae hat das Privileg in der später erweiterten Form, in der es vermutlich von Innocenz IV. am 5. September 1243 verliehen wurde. ${ }^{9}$

Quieti vestrae für dieselben, zuerst von Honorius III. am 7. März $1225^{10}$, dann in erweiterter Form, dem Liber cancellariae entsprechend, von Gregor IX. am 9. April $1228^{11}$ und von Immocenz IV. am 6. April 1244 ausgestellt. ${ }^{12}$

${ }^{1}$ Strehlke, Tabulae ordin. Theut. Nr. 309. $\quad{ }^{2}$ Strehlke Nr. $416 . \quad{ }^{3}$ Ripoll, Bullarium ordin. praedicator. Rom. 1729. I, 24. ${ }^{4}$ Ripoll I, 27. ${ }^{5}$ Ripoll

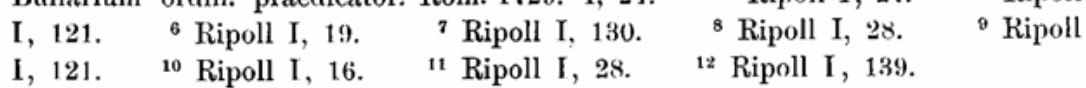


Cum dilectorum filiorum für dieselben, zuerst am 3. August 1227 von Gregor IX. ${ }^{1}$, dann von Innocenz IV. am 25̃. März 1244 verliehen. ${ }^{2}$

Quoniam habundavit iniquitas für dieselben (S. 108) wurde zuerst von Honorius III. am 18. Januar $1221^{3}$ ausgestellt, dann von Gregor IX. am 10. Mai 1227 erweitert $^{4}$ und nochmals von Innocenz IV. am 24. März 1244 verändert. ${ }^{5}$ Mit der von Innocenz IV. gewählten Form stimmt der Wortlaut des Liber cancellariae überein.

Auctoritate vobis praesentium (S. 109) für dieselben von Gregor IX. am 18. Juni $1227^{\circ}$ und gleichlautend von Innocenz IV. am 5. September 1243 gegeben. $^{7}$

Postulastis a nobis (S. 110) für dieselben von Honorius III. am 6. Mai 1221 verliehen. ${ }^{8}$ Mit demselben Wortlaut von Innocenz IV. am 4. September 1243 wiederholt. ${ }^{9}$

Cum iam per eius gratiam für dieselben. Zuerst gegeben von Innocenz IV. am 21. Januar 1244. ${ }^{10}$

In iure canonico für dieselben. Zuerst ausgestellt von Innocenz IV. am 6. April 1244. ${ }^{11}$

Quia confusio habitus für dieselben (S. 111). Zuerst verliehen von Innocenz IV. am 25. März $1244 .^{12}$

Quia confusio habitus für dieselben, gerichtet an Erzbischöfe und Bischöfe, zuerst von Gregor IX. am 25. Oktober $1239^{13}$ verliehen, dann erweitert von Innocenz IV. am 25. März 1244. ${ }^{14}$ In der letzteren Form hat das Privilegium in dem Liber cancellariae Aufnalime gefunden.

Bei den folgenden für die Predigermönche ausgestellten Urkunden waltet immer dasselbe Verhältnis ob. Sie sind teils von Gregor IX. gegeben und danu von Innocenz IV. in derselben Form oder mit geringfügigen Änderungen wiederholt, teils erst von Innocenz IV. verliehen worden. In jedem Falle stimmt der Wortlaut der in den Liber cancellariae aufgenommenen Privilegien immer mit dem von Innocenz IV. gewählten Wortlaut überein.

Über Innocenz IV. hinaus gehen, soweit wir sehen, die Änderungen nirgends.

So ist z. B. das Privilegium Licet ad hoc (S. 113) von Innocenz IV. am 6. April 1244, gegeben ${ }^{15}$, von demselben am 27. Juli

${ }^{1}$ Ripoll I, $23 . \quad{ }^{2}$ Ripoll I, 138. $\quad{ }^{3}$ Ripoll I, 11. + Ripoll I, 19. ${ }^{5}$ Ripoll I, 137. ${ }^{6}$ Ripoll I, 20. ${ }^{7}$ Ripoll I, 122. ${ }^{8}$ Ripoll I, $14 .{ }^{9}$ Ripoll I, 121. ${ }^{10}$ Ripoll I, 130. ${ }^{11}$ Ripoll I, 140. ${ }^{12}$ Ripoll I, 138. ${ }^{13} \mathrm{Ri}-$ poll I, 107. 14 Ripoll I, 138. ${ }^{15}$ Ripoll I, 139. 
1246 durch einen geringen Zusatz erweitert ${ }^{1}$, dann nochmals von Alexander IV. am 18. März $1258^{2}$ und von Klemens IV. am 11. Mai 1265 wesentlich verändert worden. ${ }^{3}$ Der Liber cancellariae ist bei der ursprünglichen von Innocenz IV. gewählten Form stehen geblieben. So erscheint auch das Privilegium Coelestis amor (S. 117), welches von Klemens IV. wesentlich erweitert wurde", im Liber cancellariae in der Gestalt, die ihm Innocenz IV. am 22. März 1244 gab. $^{5}$

Nach alledem möchten wir annehmen, daß die Sammlung im Beginn von Innocenz' IV. Pontifikat abgefaßt wurde. Besonderes Gewicht erhält diese Annahme noch dadurch, daß den im Wortlaute mitgeteilten Privilegien noch eine Reihe von Nachträgen folgen, welche über Verleihung der nämlichen Privilegien an andere religiöse Gemeinschaften berichten. Diese Nachträge beginnen mit dem Pontifikat Innocenz' IV. und gehen bis zum Beginn von dem Klemens' IV.

Mit dem Provinciale, welches außer dem Verzeichnisse der Diöcesen eine Sammlung von Privilegien umfaßte und um die Mitte des 13. Jahrhunderts bereits abgeschlossen war, ist in der Folge allerhand Verschiedenartiges, das für die Kanzlei von Wichtigkeit war, ohne bestimmte Reihenfolge vereinigt worden.

Hierher gehören zunächst die noch aus Innocenz' IV. Pontitikat herstammenden Constitutiones factae in concilio Lugdunensi von 1245 (S. 130), ferner die auf die Ämter des Vicekanzlers und der Notare bezüglichen Bestimmungen (S. 134), die Forma rescriptorum introducta ex antiquo von 1278, die aus der Zeit Bonifacius' VIII. stammende Exkommunikationssentenz über solche, welche an der Kurie Bestechung anwenden oder annehmen (S. 148), eine Bestimmung über die Abbreviatoren (S. 148), die Sanctio gloriosissimi Frederici imperatoris pro ecclesiastica libertate vom 22. Nov. 1220 (S. 149), die Bulle Honorius' III. vom 4. Januar 1221 und der durch Schriftstücke anderer Art hiervon getrennte Abschnitt Regimen et iura cancellariae more antiquo (S. 168).

Den gesammten bisher behandelten Stoff fand Merkel in einer Bologneser Handschrift des 14. Jahrh. vereint. Er schlob aus dem Charakter der Schriftstücke, daß sie für den Zweck der Kurie zusammengestellt sein müBten, und meinte, daß die ihm vorliegende Handschrift zu Studienzwecken nach Bologna gekommen sei.

\footnotetext{
' Ripoll I, 168. $\quad{ }^{2}$ Ripoll 1, 359. $\quad{ }^{3}$ Kipoll I, 451. $\quad$ 4 Ripoll I, 455.

5 Ripoll I, 136.
} 
Diese Bologneser Handschrift, aus welcher Merkel mehrere Abschnitte drucken ließ, geht auf denselben Liber cancellariae zurück, aus welchem das Kanzleibuch vom Jahre 1380 abgeschrieben wurde.

Der Liber cancellariae, welcher Dietrich von Nieheim als Vorlage diente, hat jedoch in der Folge wieder allerhand Zusätze erfahren.

Zunächst wurden einige Bestimmungen nachgetragen, welche der Vicekanzler Wilhelm, magister scholarum Parmensium, auf Befehl Papst Alexanders [IV. 1254-1261] über das Amt der Skriptoren traf.

Alsdann folgten die wichtigen Konstitutionen Johannes XXII.: (S. 157) Ratio iuris exigit, (S. 172) Paterfamilias und (S. 191) Qui exacti temporis vom 16. November 1331, die Reservationen Johanns XXII. und zwei andere Bestimmungen desselben Papstes von 1317 und (S. 196) die Konstitution Benedikts XII. vom 27. Oktober 1340 .

Endlich wurden in der Zeit Urbans VI. noch einige andere Schriftstïcke in den Liber cancellariae aufgenommen. Hierher gehört vor allem die Eidesformel, welche während des Schismas von den neugewählten Bischöfen und Äbten geleistet werden sollte (S. 156), ferner die Eidesformeln in Beginn des Liber cancellariae. Genannt wird nämlich Urban VI. in Eide des Vicekanzlers (S. 1), des Inquisitors (S. 11) und in dem nach Beendigung des Buches hinzugefügtem Eide der Poenitentiarii (S. 13). In der Eidesformel, welche bei der Übernahme des Tabellionats zu leisten war (S. 10), ist der Name Urbans wohl ausgefallen. Die Eide der Skriptoren, Abbreviatoren, Auditoren und Notarii sacri palatii sind den am Ende des Buches stehenden Konstitutionen Johannes XXII. entnommen und nur insofern geändert, als bei Erwähnung der zu beobachtenden Taxen Johanns als eines Verstorbenen gedacht wird. Auch die übrigen Eidesformeln entstammen wohl sämtlich dem 14. Jahrhundert. In denen der Rescribendarii de iustitia und de gratia wird wenigstens ausdrücklich die Taxordnung Johauns XXII. erwähnt. Älter ist vermutlich nur das Iuramentum scriptorum, qui solebant distribuere litteras rescribendas cancellariae duntaxat, auf S. 3, das als antiquum bezeichnet wird.

Der Stilus palatii abbreviatus Dietrichs von Nieheim findet sich in dem Cod. lat. 3063 der Königlichen Bibliothek zu München.

Die Handschrift stammt aus dem 15. Jahrhundert und gehörte, einem Eintrag auf der Innenseite des Vorderdeckels zufolge, ur- 
sprünglich dem egregius doctor dominus Georius Dittolf, Kanzler des Herzogs Wilhelm, der sie dem Kloster Andechs zum Geschenk darbrachte. Sie enthält zumeist kirchenrechtliche Sachen, z. B. das Repertorium antiquarum decisionum secundum rubricas decretorum compositum per quondam Arvassanum utriusque iuris doctorem auditorem causarum. ${ }^{2}$ Von Blatt 18 an sind die Festtage des Palatium verzeichnet und finden sich im AnschluB an diese kurze Aufzeichnungen, umfassend die Zeit von Martins V. Wahl bis zum 28. August 1421, zur Rückkehr des Papstes von Tivoli nach Rom. Dann folgen die handschriftlich sehr viel verbreiteten Conclusiones sive determinationes ... quorundam dubiorum, in quibus finaliter omnes vel saltim maior pars dominorum [scil. coauditorum] praedictorum et aliorum postea superveniencium remanserunt, die der Auditor Wilhelm von Horborg vom 30. Januar 1376 bis Mai 1386 sammelte, und die Decisiones rotae antiquae, welche beiden letztgenannten Werke von Petrus Schoyffer de Gernssheym 1477 in Mainz gedruckt worden sind. Den Schluß bilden ein Stilus secundum simplicem practicam sacri palatii causarum und das Summarium decisionum domini Egidii de Bellemere.

Verschiedene Hände haben an dieser Sammlung geschrieben.

In diesem Sammelbande findet sich auf fünf Blättern, welche oben in der Mitte die alten Nummern CCXXVIII bis CCXXXII tragen, Dietrichs Schrift. Rechts oben am Rande des ersten Blattes ist die neue Folionummer 254 eingetragen. Die folgendẹn Blätter haben jedoch keine Nummer erhalten. Von diesen Blättern, welche $23,8 \mathrm{~cm}$ hoch und $22 \mathrm{~cm}$ breit sind, sind die ersten drei Papier-, die beiden letzten Pergamentblätter.

Zwei von einander verschiedene Hände wechseln in dem Schriftchen ab.

Der Text von Dietrichs Schrift beginnt mit den Worten: In nomine domini amen. Salva meliori practica sequitur stilus palatii abbreviatus et conceptus ex communi practica dicti palatii per T. de Nyem, abbreviatorem litterarum apostolicarum minimum.

Am Rande des Blattes, oberhalb dieser Worte, hat dieselbe Hand bemerkt: Stilus curie Romane servandus est c. Venerabiles, De confirmatione utili vel inutili ${ }^{2}$ et c. Quam gravi, De crimine falsi. ${ }^{3}$ Während die Werke Wilhelms von Horborg und Egidius' de

${ }^{1}$ Wurde 1391 von Bonifacius IX. zum Bischof von Paderborn ernannt. Vgl. des Herausgebers Dietrich von Nieheim \$. 109. ' ' Deeret Greg. IX. Iih. II. Tit. XXX. c. $8 .{ }^{3}$ Decret Greg. IX. Lib. V. 'Tit. XX. c. 6. 
Bellemere mehr ein juristisches Interesse verfolgen, hat Dietrich von Nieheim in seinem Stilus vor allem den Standpunkt des in der Rota thätigen Kanzleibeamten im Auge und behandelt von diesem Standpunkte aus den an der Rota üblicheu Rechtsgang, von der Instruktion des Prozesses an bis zum Urteilsspruch. Das übersichtlich geordnete und klar geschriebene Werkchen sollte wohl der Belehrung für die bei der Rota eintretenden Notare, Advokaten, Prokuratoren und Auditoren dienen, mußte aber auch für alle diejenigen von Interesse sein, welche an diesem Gerichtshofe einen Prozeß zu führen hatten.

Das Werkchen mag sich großer Verbreitung erfreut und Dietrichs Namen bekannt gemacht haben. Als im Jahre 1471 ein Schreiber, welcher die unter Dietrichs Namen von Eccard herausgegebene Papstchronik kopiert hatte, des Autors gedachte, den er fälschlich als Johannes de Nyunheym bezeichnet, nennt er ihn auch primus inventor stili cancellariae. ${ }^{1}$ Aller Wahrscheinlichkeit nach bezieht sich diese freilich nicht ganz genaue Angabe auf den Stilus abbreviatus sacri palatii.

Das Schriftchen zerfällt in folgende T'eile:

De causarum commissionibus . . . . . . . . . . . 217

De observationibus terminorum . . . . . . . . . . . . 218

De decreto citationis ad partes aut commissione per partium praesentiam praesentata . . . . . . . . . . . 218

De observationibus aliorum terminorum . . . . . . . 220

De admissione articulorum . . . . . . . . . . . . 221

De remissione ad partes decretid . . . . . . . . . . 222

De modo servando in tradendo litteram remissionis . . . 223

De terminis ad audiendum voluntatem domini super admissione articulorum . . . . . . . . . . . . . . . . 223

De iuribus aut testibus productis post conclusionem . . . 224

De testibus affuturis . . . . . . . . . . . . . . 224

De receptione testium affuturorum . . . . . . . . . 224

De conclusionibus . . . . . . . . . . . . . . . 225

De litis contestatione . . . . . . . . . . . . . . 225

De compulsione partis ad respondendum positionibus . . . 226

De iuramento calumniae . . . . . . . . . . . . . 226

De secunda et tertia instantijs . . . . . . . . . . . . . 226

${ }^{1}$ Handschr. der Hofbibl. zu Wien Nr. 3331 chart. fol. XV. 
De emergentibus causarum in gravaminibus et terminis observandis in eisdem . . . . . . . . . . . . . . 227

Quod causae ad palatium deductae non remittantur . . . 228

De absolutione ad cautelam et simpliciter . . . . . . . 228

De appellationibus interponendis . . . . . . . . . . . 228

De causis spoliationum et suspensione petitorii . . . . . 229

De suspensione petitorii . . . . . . . . . . . . , 229

De resumptione petitorii . . . . . . . . . . . . . . 230

De non resumptione petitorii et attemptatorum . . . . . 230

De observatione terminorum in possessorio in omnibus instantijs 230

De attemptatis et modo, quando lite pendente . . . . . 231

De terminis super attemptatis observandis . . . . . . . 231

De restitutione in integrum . . . . . . . . . . . . 232

De inhibitione . . . . . . . . . . . . . . . . 232

De processibus super desertionibus appellationum . . . . 232

Super nullitate processuum . . . . . . . . . . . . 233

De taxatione expensarum . . . . . . . . . . . . . 233

De auditoribus . . . . . . . . . . . . . . . . 234

Noch bleibt die Frage zu erörtern, wann Dietrich von Nieheim das Schriftchen verfaßte.

Der Ausdruck, den er S. 220 gebraucht: Et id idem per aliud tempus domino nostro Ur[bano] VI. moderno sedi apostolice presidente vidi servari, et in palatio predicto tunc eciam me adhuc dicti palacii notario existente beweist zunächst, daß Dietrich seine Schrift unterm Pontifikat Urbans VI., also zwischen 1378 und 1389 abfaßte.

Eine nähere Bestimmung ist vielleicht noch möglich. Dietrich nennt sich im Eingange zu seiner Schrift Abbreviator. Dieses Amt allein könnte er in den ersten Wochen Urbans VI. kurze Zeit bekleidet haben, wiewohl er noch im Anfang von Urbans VI. Pontifikat, seinen eigenen oben angeführten Worten nach, Notar war und schon am 11. Mai 1378, also zu Beginn der vierten Woche Urbans VI., als Skriptor nachzuweisen ist. ${ }^{1}$ Da $B$ aber in jenen Tagen das Schriftchen niedergeschrieben sein könnte, wird durch einige andere Ausdrücke des. Verfassers widerlegt. Einmal sagt er auf S. 219: quod non multis retroactis temporibus in rota observari consuevit et me eciam licet indigno tunc aliquamdiu notario dicti palacij existente. Der

${ }^{1} \mathrm{~s}$. des Herausgebers Dictrich von Nieheim S. 26. 
Ausdruck „non multis retroactis temporibus“ würde von Dietrich nicht gewählt worden sein, wenn er 8-14 Tage vorher noch der Rota angehörte. Daß mehrere Jahre wenigstens seit Dietrichs Austritt aus dem Gerichtshofe vergangen waren, beweist schlagender noch die Bemerkung, die er zu einem Gebrauche der Rota macht: "Quid autem hodie in talibus servetur ignoro, credo tamen idem, ut supra, servari.“

$\mathrm{DaB}$ freilich in den späteren Jahren Urbans VI. Dietrich eine Zeit lang nur Abbreviator gewesen sei, läßt sich nicht beweisen, nur vermuten.

Dietrich hatte im Jahre 1385 den Papst in Nocera verlassen und war zu dessen Feind, König Karl von Neapel, gegangen. Erst im Jahre 1387 war er an die Kurie nach Lucca zurückgekehrt. Die Vermutung liegt daher nahe, daß er wegen seiner Flucht die Ämter eines Skriptors und Abbreviators, die er bis dahin bekleidet hatte, einbüßte und erst nach der Rückkehr an die Kurie und der Wiederaufnahme durch Urban die Abbreviatorie und nach kurzer Zeit auch wieder das Amt eines Skriptors erlangte.

Während jener Zeit, in der er nur Abbreviator war, müBte er demnach den Stilus geschrieben haben.

Zu ehrerbietigem Danke fühlt sich der Herausgeber Sr. Excellenz. dem Herrn Staatsminister Dr. von Gerber verpflichtet, durch dessen gütige Vermittelung es ihm gestattet war, die Pariser Handschrift des Liber cancellariae in Leipzig benutzen zu dürfen. Den Leitern der Königl. Hof- und Staatsbibliothek zu München und der Bibliothèque nationale $\mathrm{zn}$ Paris, welche ihm die seiner Ausgabe zu Grunde gelegten Handschriften zur Benutzung an seinem Wohnorte überließen, spricht er auch an dieser. Stelle seinen aufrichtigsten Dank aus.

\section{INHAL'T.}

Der Liber cancellariae apostolicae vom Jahre $1380 \cdot$ Seite

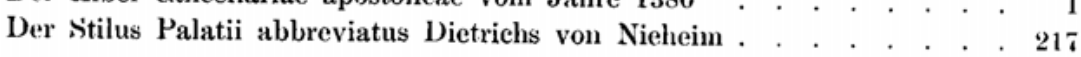

